\title{
X-Ray Vision with Only WiFi Power Measurements Using Rytov Wave Models
}

\author{
Saandeep Depatla, Lucas Buckland and Yasamin Mostofi
}

\begin{abstract}
In this paper, unmanned vehicles are tasked with seeing a completely unknown area behind thick walls based on only wireless power measurements using WLAN cards. We show that a proper modeling of wave propagation that considers scattering and other propagation phenomena can result in a considerable improvement in see-through imaging. More specifically, we develop a theoretical and experimental framework for this problem based on Rytov wave models, and integrate it with sparse signal processing and robotic path planning. Our experimental results show high-resolution imaging of three different areas, validating the proposed framework. Moreover, they show considerable performance improvement over the state-of-the-art that only considers the Line Of Sight (LOS) path, allowing us to image more complex areas not possible before. Finally, we show the impact of robot positioning and antenna alignment errors on our see-through imaging framework.
\end{abstract}

\section{INTRODUCTION}

Passive device-free localization and mapping of objects in an environment has recently received considerable attention. There are several potential applications for such approaches, from location-aware services, to search and rescue, and robotic networks.

A survey of the related literature indicates that localization and mapping has been investigated by three different communities. More specifically, in the networking community, both devicebased and device-free localization based on RF signals have been explored, typically in the context of tracking human motion [1]-[7]. However, in most these setups, either the object of

(C)2013 IEEE. Personal use of this material is permitted. However, permission to use this material for any other purposes must be obtained from the IEEE by sending a request to pubs-permissions@iee.org.

The authors are with the Department of Electrical and Computer Engineering, University of California, Santa Barbara, CA 93106, USA $\{$ saandeep, lbuckland, ymostofi\}@ece.ucsb.edu. 
interest is not occluded or the information of the first layer of occluder is assumed known. Furthermore, most focus has been on motion tracking and not on high-resolution imaging. In robotics, localization and mapping of objects is crucial to proper navigation. As such, several work, such as Simultaneous Localization and Mapping (SLAM), has been developed for mapping based on laser scanner measurements [8]-[11]. However, in these approaches, mapping of occluded objects is not possible. For instance, in [12], some information of the occluded objects is first obtained with radar and then utilized as part of robotic SLAM.

In the electromagnetic community, there has been interest in solving an inverse scattering problem [13], i.e., deducing information about objects in an environment based on their impact on a transmitted electromagnetic wave [14]-[16]. For instance, remote sensing to detect oil reserves beneath the surface of the earth is one example [17]. Traditional medical imaging based on X-ray also falls into this category [13]. There has also been a number of work on using a very general wave propagation model for inverse scattering, such as Distorted Born Iterative method [14], contrast source inversion method [18], and stochastic methods [19]. However, the computational complexity of these approaches makes it prohibitive for high-resolution imaging of an area of a reasonable size. Furthermore, most such approaches utilize bulky equipments, which makes their applicability limited.

In this paper, we are interested in high-resolution see-through imaging of a completely unknown area, based on only WiFi measurements, and its automation with unmanned vehicles. With the advent of WiFi signals everywhere, this sensing approach would make our framework applicable to several indoor or outdoor scenarios. The use of robotic platforms further allows for autonomous imaging. However, the overall problem of developing an autonomous system that can see everything through walls is considerably challenging due to three main factors: 1) proper wave modeling is crucial but challenging; 2) the resulting problem is severely under-determined, i.e., the number of measurements typically amount to only a few percentage of the number of unknowns; and 3) robot positioning is prone to error, adding additional source of uncertainty to the imaging. In our past work [20], [21], we have shown that seeing through walls and imaging a completely unknown area is possible with only WiFi signals. However, we only considered the Line of Sight (LOS) path and the impact of the objects along this path when modeling the receptions. A transmitted WiFi signal will experience several other propagation phenomena such as scattering that an LOS model can not embrace. This can then result in a significant gap 
between the true receptions (RF sensed values) and the way they were modelled (see Fig. 7 for instance), and is one of the main bottlenecks of the state of the art in RF sensing.

Thus, the first contribution of this paper is to address this bottleneck and enable see-through imaging of more complex areas not possible before. In order to do so, we have to come up with a way of better modeling the receptions to achieve a leap in the imaging results, while maintaining a similar computational complexity. If we take the modeling approaches of the communication/networking literature, the term multipath is typically used to describe propagation phenomena beyond the LOS path. However, the modeling of multipath in these literature, which is done via probabilistic approaches or ray tracing, is not suitable for high-resolution detailed imaging through walls. In this paper, we then tap into the wave propagation literature to model the induced electric field over the whole area of interest and include the impact of objects that are not directly on the LOS path. While Maxwell's equations can accurately model the RF sensed values, it is simply not feasible to start with that level of modeling. A further tapping into the wave literature then shows several possible approximations to Maxwell's equations. In this paper, we show that modeling the receptions based on Rytov wave approximation can make a significant improvement in the see-through imaging results. Rytov approximation is a linearizing wave approximation model, which also includes scattering effects [13]. While it has been discussed in the electromagnetic literature in the context of inverse scattering [13], [22], [23], there are no experimental results that show its performance for see through imaging, especially at WiFi frequencies. In this paper, it is therefore our goal to build on our previous work and significantly extend our sensing model based on Rytov wave approximation.

The second contribution of this paper is on achieving a higher level of automation. More specifically, in [24], the two robots had to constantly coordinate their positioning and antenna alignment, and their positioning errors were manually corrected several times in a route (e.g. every $1 \mathrm{~m}$ ). In this paper, each robot travels a route (see definition in Section IV) autonomously and without any coordination with the other robot or positioning error correction. It is therefore feasible to collect measurements much faster, reducing the experiment time from several hours to a few minutes. However, this comes at the cost of non-negligible errors in robot positioning and antenna alignment, the impact of which we discuss and experimentally validate in Section $\mathrm{V}$.

Finally, the last contribution of this paper is to show that two robots can see through walls 
and image an area with a higher level of complexity, which was not possible before. More specifically, we integrate modeling of the receptions, based on Rytov approximation, with sparse signal processing (utilized in our past work for RF imaging) and robotic path planning and show how a considerable improvement in imaging quality can be achieved. We experimentally validate this by successfully imaging three different areas, one of which can not be imaged with the state of the art while the two others show a considerable improvement in imaging quality.

The rest of the paper is organized as follows. In Section II, we mathematically formulate our imaging problem based on Rytov wave approximation. In Section III, we pose the resulting optimization problems and discuss how to solve them based on total variation minimization. In Section IV, we introduce the hardware and software structures of our current experimental robotic platform, which allows for more autonomy in WiFi measurement collection. In Section $\mathrm{V}$, we then present our imaging results of three different areas and show the impact of robot positioning and antenna alignment errors. We conclude in Section VI.

\section{Problem Formulation}

Consider a completely unknown workspace $\mathbf{D} \subset \mathbb{R}^{3}$. Let $\mathbf{D}^{\text {out }}$ be the complement of $\mathbf{D}$, i.e., $\mathbf{D}^{\text {out }}=\mathbb{R}^{3} \backslash \mathbf{D}$. We consider a scenario where a group of robots in $\mathbf{D}^{\text {out }}$ are tasked with imaging the area $\mathbf{D}$ by using only WiFi. In other words, the goal is to reconstruct $\mathbf{D}$, i.e., to determine the shapes and locations of the objects in $\mathbf{D}$ based on only a small number of WiFi measurements. We are furthermore interested in see-through imaging, i.e., the area of interest can have several occluded parts, like parts completely behind concrete walls and thus invisible to any node outside. Fig. 1 shows an example of our considered scenario. The red superimposed volume marks the area that the two unmanned vehicles are interested in imaging but that is completely unknown to them. The area has several occluded parts, such as the parts blocked by the outer concrete wall, which is highly attenuating. Note that both empty and full spaces inside the red volume as well as its outer surfaces are all unknown to the robots and need to be imaged. The robots only have WiFi for imaging. As the robots move outside of $\mathbf{D}$, one robot measures the received signal power from the transmissions of the other robot. The unknown area $\mathbf{D}$ then interacts with each transmission, as dictated by the locations and properties of its objects, leaving its impact on each reception. The robots then need to image the structure based on all the receptions. 


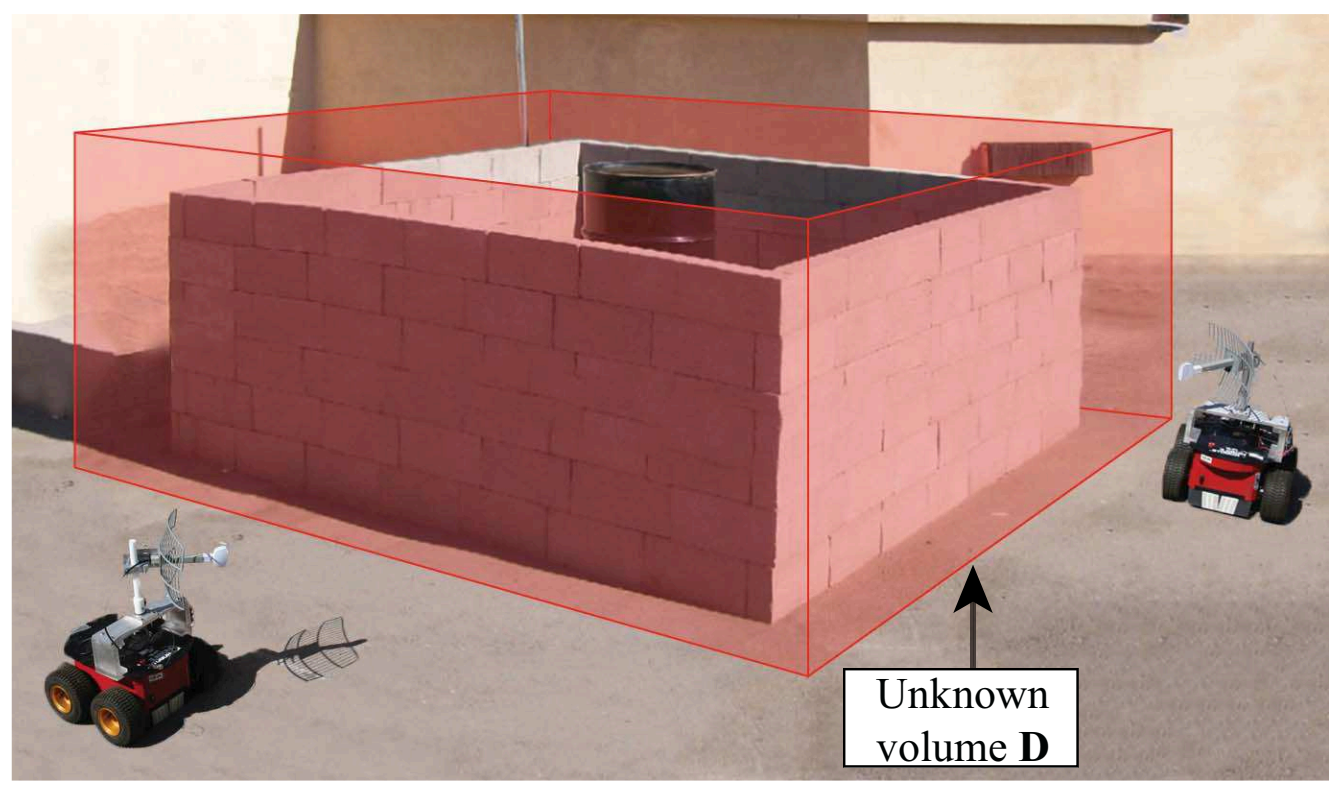

Fig. 1. Two robots are tasked with imaging the unknown area $\mathbf{D}$ that is marked with the red superimposed volume, which involves seeing through walls, based on only a small number of WiFi measurements. Note that this figure is generated for illustrative purposes. For a true snapshot of the robots in operation, see Fig. 9.

In this section, we start with the volume integral wave equation and discuss how it can be linearized and solved under certain assumptions, developing the system models that we shall utilize later for our imaging. The readers are referred to [13], [25] for more details on the wave propagation modeling.

\section{A. Volume Integral Equations [13]}

Let $\mathbf{E}(\mathbf{r})$ be the electric field, $\mathbf{J}(\mathbf{r})$ be the current density, $\epsilon(\mathbf{r})$ be the electric permittivity, and $\mu(\mathbf{r})$ be the magnetic permeability at $\mathbf{r} \in \mathbb{R}^{3}$, where $\mathbf{r}$ is the position vector in the spherical coordinates. ${ }^{1}$ Then, we have the following volume integral equation relating the electric field to the current source and objects in D [13]

$$
\mathbf{E}(\mathbf{r})=j \omega \mu_{0} \iiint_{\mathbb{R}^{3}} \overrightarrow{\mathbf{G}}\left(\mathbf{r}, \mathbf{r}^{\prime}\right) \bullet \mathbf{J}\left(\mathbf{r}^{\prime}\right) d v^{\prime}+\iiint_{\mathbb{R}^{3}} \overrightarrow{\mathbf{G}}\left(\mathbf{r}, \mathbf{r}^{\prime}\right) \bullet\left(O\left(\mathbf{r}^{\prime}\right) \mathbf{E}\left(\mathbf{r}^{\prime}\right)\right) d v^{\prime},
$$

\footnotetext{
${ }^{1}$ Throughout the paper, single-frequency operation is assumed and all the materials are considered isotropic and non-magnetic, i.e., $\mu(\mathbf{r})=\mu_{0}$, for all $\mathbf{r} \in \mathbb{R}^{3}$, where $\mu_{0}$ is the permeability of the freespace.
} 
where $\overrightarrow{\mathbf{G}}\left(\mathbf{r}, \mathbf{r}^{\prime}\right)$ is the dyadic Green's function given by

$$
\begin{aligned}
\overrightarrow{\mathbf{G}}\left(\mathbf{r}, \mathbf{r}^{\prime}\right) & =\left(I+\frac{\nabla \nabla}{k_{0}^{2}}\right) g\left(\mathbf{r}, \mathbf{r}^{\prime}\right), \\
g\left(\mathbf{r}, \mathbf{r}^{\prime}\right) & =\frac{e^{j k_{0}\left|\mathbf{r}-\mathbf{r}^{\prime}\right|}}{4 \pi\left|\mathbf{r}-\mathbf{r}^{\prime}\right|}
\end{aligned}
$$

$O(\mathbf{r})=k^{2}(\mathbf{r})-k_{0}^{2}$ denotes the material property of the object at position $\mathbf{r}, k_{0}^{2}=\omega^{2} \epsilon_{0} \mu_{0}$ denotes the wavenumber of the free space, ${ }^{2} k^{2}(\mathbf{r})=\omega^{2} \mu_{0} \epsilon(\mathbf{r})$ denotes the wavenumber of the medium at $\mathbf{r}, \epsilon_{0}$ and $\mu_{0}$ are the permittivity and permeability of the free space respectively, $\omega$ is the angular frequency, and • denotes the vector dot product. The robots are then interested in learning $O(\mathbf{r})$, for $\mathbf{r} \in \mathbf{D}$, as it carries the information of the location/material property of the objects in the workspace. Note that (1) is valid for any inhomogeneous, isotropic, and nonmagnetic media. Also, $O(\mathbf{r}) \mathbf{E}(\mathbf{r})$ is the equivalent current induced in the object at $\mathbf{r}$. This induced current in turn produces an electric field. The total field is then the sum of the electric field due to the current in the transmit antenna, the first term on the right hand side (RHS) of (1), and the electric field due to the induced current in the objects (the second term on the RHS of (1)).

First, we start by assuming free space in $\mathbf{D}^{\text {out }}$. Then, $\epsilon(\mathbf{r})=\epsilon_{0}$, for $\mathbf{r} \in \mathbf{D}^{\text {out }}$, resulting in $k^{2}(\mathbf{r})=k_{0}^{2}$ and $O(\mathbf{r}) \equiv 0$, for $\mathbf{r} \in \mathbf{D}^{\text {out }}$. When there are no objects in $\mathbf{D}$, we have $k^{2}(\mathbf{r})=k_{0}^{2}$ and $O(\mathbf{r}) \equiv 0$, for all $\mathbf{r} \in \mathbb{R}^{3}$, and the second term on the RHS of (1) vanishes. This means that the first term is the incident field when there are no objects in D and the second term is the result of scattering from the objects in D. By denoting the first term on the RHS of (1) as $\mathbf{E}_{\text {inc }}(\mathbf{r})$, we then get

$$
\mathbf{E}(\mathbf{r})=\mathbf{E}_{\mathrm{inc}}(\mathbf{r})+\iiint_{\mathbf{D}} \overrightarrow{\mathbf{G}}\left(\mathbf{r}, \mathbf{r}^{\prime}\right) \bullet\left(O\left(\mathbf{r}^{\prime}\right) \mathbf{E}\left(\mathbf{r}^{\prime}\right)\right) d v^{\prime}
$$

where $\overrightarrow{\mathbf{G}}\left(\mathbf{r}, \mathbf{r}^{\prime}\right)$ is a second-order tensor and can be represented as the following $3 \times 3$ matrix in the Cartesian coordinates:

$$
\overrightarrow{\mathbf{G}}\left(\mathbf{r}, \mathbf{r}^{\prime}\right)=\left[\begin{array}{l}
G_{x x}\left(\mathbf{r}, \mathbf{r}^{\prime}\right) G_{x y}\left(\mathbf{r}, \mathbf{r}^{\prime}\right) G_{x z}\left(\mathbf{r}, \mathbf{r}^{\prime}\right) \\
G_{y x}\left(\mathbf{r}, \mathbf{r}^{\prime}\right) G_{y y}\left(\mathbf{r}, \mathbf{r}^{\prime}\right) G_{y z}\left(\mathbf{r}, \mathbf{r}^{\prime}\right) \\
G_{z x}\left(\mathbf{r}, \mathbf{r}^{\prime}\right) G_{z y}\left(\mathbf{r}, \mathbf{r}^{\prime}\right) G_{z z}\left(\mathbf{r}, \mathbf{r}^{\prime}\right)
\end{array}\right]
$$

${ }^{2}$ In this paper, free space refers to the case where there is no object. 
In reality, there will be objects in $\mathbf{D}^{\text {out }}$. Then, $\mathbf{E}_{\text {inc }}$ denotes the field when there are no objects in D. ${ }^{3}$ Without loss of generality, we assume that the transceiver antennas are linearly polarized in the $z$-direction. This means that we only need to calculate the $z$-component of the electric field, which depends on the last row of $\overrightarrow{\mathbf{G}}\left(\mathbf{r}, \mathbf{r}^{\prime}\right)$. Let $\mathbf{J}_{\mathrm{eq}}(\mathbf{r})=\left[\begin{array}{lll}J_{\mathrm{eq}}^{x} & J_{\mathrm{eq}}^{y} & J_{\mathrm{eq}}^{z}\end{array}\right]^{T}=O(\mathbf{r}) \mathbf{E}(\mathbf{r})$. We further assume near-zero cross-polarized components $J_{\text {eq }}^{x}$ and $J_{\text {eq }}^{y}$ and take $\mathbf{J}_{\text {eq }}(\mathbf{r}) \approx\left[\begin{array}{lll}0 & 0 & J_{\text {eq }}^{z}\end{array}\right]^{T}$. This approximation is reported to have a negligible effect [26]. By using this approximation in (4) and only taking the $z$-component, we get the following scalar equation:

$$
E^{z}(\mathbf{r})=E_{\mathrm{inc}}^{z}(\mathbf{r})+\iiint_{\mathbf{D}} G_{z z}\left(\mathbf{r}, \mathbf{r}^{\prime}\right) O\left(\mathbf{r}^{\prime}\right) E^{z}\left(\mathbf{r}^{\prime}\right) d v^{\prime},
$$

where $E^{z}(\mathbf{r})$ and $E_{\text {inc }}^{z}(\mathbf{r})$ are the z-components of $\mathbf{E}(\mathbf{r})$ and $\mathbf{E}_{\mathrm{inc}}(\mathbf{r})$, respectively.

\section{B. Linearizing Approximations}

In (5), the received electric field $E^{z}(\mathbf{r})$ is a non-linear function of the object function $O(\mathbf{r})$, since $E^{z}\left(\mathbf{r}^{\prime}\right)$ inside the integral also depends on $O(\mathbf{r})$. This nonlinearity is due to the multiple scattering effect in the object region [25]. Thus, we next use approximations that make (5) linear and easy to solve under the setting of sparse signal processing.

1) Line Of Sight-Based Modeling [13], [20]: A simple way of modeling the receptions is to only consider the LOS path from the transmitter to the receiver and the impact of the objects on this path. This model has been heavily utilized in the literature due to its simplicity [20]. However, it results in a considerable modeling gap for see-through imaging since it does not include important propagation phenomena such as scattering. In this part, we summarize the LOS model in the context of wave equations.

At very high frequencies, such as in X-ray, the wave can be assumed to travel in straight lines with negligible reflections and diffractions along its path [13]. Then, the solution to (5) is given as follows by using Wentzel Kramers Brillouin (WKB) approximation, ${ }^{4}$

$$
E(\mathbf{r})=\frac{c}{\sqrt{\alpha(\mathbf{r})}} e^{j \omega \int_{\mathbb{L}_{T \rightarrow R}} \alpha\left(\mathbf{r}^{\prime}\right) d \mathbf{r}^{\prime}}, \quad \text { WKB Approximation }
$$

\footnotetext{
${ }^{3}$ In our experiments, we will not have access to the exact incident field when there is nothing in $\mathbf{D}$. Thus, the two robots make a few measurements in $\mathbf{D}^{\text {out }}$ where there are no objects in between them to estimate and remove the impact of $\mathbf{E}_{\text {inc. }}$ If the robots have already imaged parts of $\mathbf{D}^{\text {out }}$, that knowledge can be easily incorporated to improve the performance.

${ }^{4}$ Here, the field is along the $z$-direction, as explained before. From this point on, superscript $z$ is dropped for notational convenience.
} 
where $\alpha(\mathbf{r})$ is a complex number that represents the slowness of the medium at $\mathbf{r}$ and is related to $k(\mathbf{r}), \int_{\mathbb{L}_{T \rightarrow R}}$ is a line integral along the line joining the positions of the transmitter and the receiver, and $c$ is a constant that depends on the transmitted signal power.

It can be seen that the loss incurred by the ray is linearly related to the objects along that path, resulting in a linear relationship between the received power and the objects, as we shall see. This approximation is the base for X-ray tomography [27]. However, the underlying assumption of this method is not valid at lower frequencies, like microwave frequencies, due to the nonnegligible diffraction effects [28]. In [20], [21], we proposed a see-through wall RF-based imaging framework based on this approximation. In this paper, our goal is use a considerably more comprehensive modeling of the receptions (which has been a bottleneck in see-through imaging) by tapping into the wave literature. We show that by addressing the modeling of the receptions through using Rytov wave approximation, we can image areas not possible before.

2) Rytov Approximation [13]: In general, the field inside any inhomogeneous media can be expressed as

$$
E(\mathbf{r})=e^{j \psi(\mathbf{r})}
$$

and satisfies

$$
\left[\nabla^{2}+k^{2}(\mathbf{r})\right] E(\mathbf{r})=0
$$

where $\psi(\mathbf{r})$ is a complex phase term. It can then be shown that the solution to (8) can be approximated as follows:

$$
E(\mathbf{r})=E_{\text {inc }}(\mathbf{r}) e^{j \phi(\mathbf{r})}, \quad \text { Rytov Approximation }
$$

where

$$
\phi(\mathbf{r})=\frac{-j}{E_{\text {inc }}(\mathbf{r})} \iiint_{\mathbf{D}} g\left(\mathbf{r}, \mathbf{r}^{\prime}\right) O\left(\mathbf{r}^{\prime}\right) E_{\text {inc }}\left(\mathbf{r}^{\prime}\right) d v^{\prime} .
$$

The validity of Rytov approximation is established by dimensional analysis in [13] and is accurate at high frequencies, ${ }^{5}$ if

$$
\delta_{\epsilon}(\mathbf{r}) \triangleq \frac{\epsilon(\mathbf{r})}{\epsilon_{0}}-1 \ll 1
$$

\footnotetext{
${ }^{5}$ Throught this paper, high frequency refers to the frequencies at which the size of inhomogeneity of objects is much larger than the wavelength.
} 
where $\delta_{\epsilon}(\mathbf{r})$ is the normalized deviation of the electric permittivity from the free space. At lower frequencies, the condition for validity of the Rytov approximation becomes

$$
\left(k_{0} L\right)^{2} \delta_{\epsilon}(\mathbf{r}) \ll 1
$$

where $L$ is the order of the dimension of the objects. In our case, with a frequency of $2.4 \mathrm{GHz}$ and $L$ of the order of $1 \mathrm{~m}$, we satisfy the condition of high frequency, except at the boundaries of the objects, where there are abrupt changes in the material.

For the sake of completion, a more commonly-used linearizing approximation, called Born approximation, is summarized in the appendix. Rytov approximation is reported to be more relaxed than the Born approximation at higher frequencies [13]. Also, Rytov approximation lends itself to a simple linear form, when we only know the magnitude of the received electric field, as described next. Thus, in this paper, we focus on Rytov wave modeling.

\section{Intensity-Only Rytov Approximation}

In the aforementioned equations, both magnitude and phase of the received field are needed. In this paper, however, we are interested in imaging based on only the received signal power.

Then, by conjugating (9), we get

$$
E^{*}(\mathbf{r})=E_{\mathrm{inc}}^{*}(\mathbf{r}) e^{-j \phi^{*}(\mathbf{r})}
$$

From (9) and (11), we then have

$$
|E(\mathbf{r})|^{2}=\left|E_{\text {inc }}(\mathbf{r})\right|^{2} e^{-2 \operatorname{Imag}(\phi(\mathbf{r}))},
$$

where $\operatorname{Imag}($.$) and |$.$| denote the imaginary part and the magnitude of the argument, respectively.$ Since the received power ${ }^{6}$ is proportional to the square of the magnitude of the received field, we have the following equation by taking logarithms on both sides of (12):

$$
P_{\mathrm{r}}(\mathbf{r})(\mathrm{dBm})=P_{\mathrm{inc}}(\mathbf{r})(\mathrm{dBm})+10 \log _{10}\left(e^{-2}\right) \operatorname{Imag}(\phi(\mathbf{r})),
$$

where $P_{\mathrm{r}}(\mathbf{r})(\mathrm{dBm})=10 \log _{10}\left(\frac{|E(\mathbf{r})|^{2}}{120 \pi \times 10^{-3}}\right)$ is the received power in $\mathrm{dBm}$ at $\mathbf{r}$, and $P_{\mathrm{inc}}(\mathbf{r})(\mathrm{dBm})=$ $10 \log _{10}\left(\frac{\left|E_{\text {inc }}(\mathbf{r})\right|^{2}}{120 \pi \times 10^{-3}}\right)$ is the power incident in $\mathrm{dBm}$ at $\mathbf{r}$ when there are no objects.

${ }^{6}$ This is the received power by an isotropic antenna. For a directional antenna, this should be multiplied by the gain of the antenna. 
To solve (12) for object $O(\mathbf{r})$, we discretize $\mathbf{D}$ into $N$ equal-volume cubic cells. The position of each cell is represented by its center position vector $\mathbf{r}_{n}$, for $n \in\{1,2, \cdots, N\}$. The electric field and the object properties are assumed to be constant within each cell. We then have

$$
\begin{gathered}
O(\mathbf{r})=\sum_{n=1}^{N} O\left(\mathbf{r}_{n}\right) C_{n}, \\
E_{\text {inc }}(\mathbf{r})=\sum_{n=1}^{N} E_{\mathrm{inc}}\left(\mathbf{r}_{n}\right) C_{n},
\end{gathered}
$$

where $\mathbf{r}, \mathbf{r}_{n} \in \mathbf{D}, C_{n}$ is a pulse basis function which is one inside cell $n$ and zero outside. By substituting (14) and (15) into (10), we get

$$
\begin{aligned}
\phi(\mathbf{r}) & =\frac{-j}{E_{\text {inc }}(\mathbf{r})} \sum_{n=1}^{N} O\left(\mathbf{r}_{n}\right) E_{\mathrm{inc}}\left(\mathbf{r}_{n}\right) \iiint_{V_{n}} g\left(\mathbf{r}, \mathbf{r}^{\prime}\right) d v^{\prime} \\
& \approx \frac{-j}{E_{\text {inc }}(\mathbf{r})} \sum_{n=1}^{N} g\left(\mathbf{r}, \mathbf{r}_{n}\right) O\left(\mathbf{r}_{n}\right) E_{\text {inc }}\left(\mathbf{r}_{n}\right) \Delta V,
\end{aligned}
$$

where

$$
\iiint_{V_{n}} g\left(\mathbf{r}, \mathbf{r}^{\prime}\right) d v^{\prime} \approx g\left(\mathbf{r}, \mathbf{r}_{n}\right) \Delta V,
$$

$V_{n}$ is the $n^{\text {th }}$ cell and $\Delta V$ is the volume of each cell. Note that $C_{n}$ is not included in (16) since we are evaluating the integral inside cell $n$ where $C_{n}$ is one.

Let $\left(\mathbf{p}_{i}, \mathbf{q}_{i}\right)$, for $\mathbf{p}_{i}, \mathbf{q}_{i} \in \mathbf{D}^{\text {out }}$, denote the transmitter and receiver position pair where the $i^{\text {th }}$ measurement is taken. Also, let $\Phi=\left[\phi_{\mathbf{p}_{1}}\left(\mathbf{q}_{1}\right) \phi_{\mathbf{p}_{2}}\left(\mathbf{q}_{2}\right) \cdots \phi_{\mathbf{p}_{M}}\left(\mathbf{q}_{M}\right)\right]^{T}$, where $M$ is the number of measurements, $\phi_{\mathbf{p}_{i}}\left(\mathbf{q}_{i}\right)=\frac{-j}{E_{\text {inc, } \mathbf{p}_{i}}\left(\mathbf{q}_{i}\right)} \sum_{n=1}^{N} g\left(\mathbf{q}_{i}, \mathbf{r}_{n}\right) O\left(\mathbf{r}_{n}\right) E_{\mathrm{inc}, \mathbf{p}_{i}}\left(\mathbf{r}_{n}\right) \Delta V$, and $E_{\text {inc, } \mathbf{p}_{i}}\left(\mathbf{r}_{n}\right)$ is the incident field at $\mathbf{r}_{n}$ when the transmitter is at $\mathbf{p}_{i}$. Then, we have

$$
\Phi=-j F O,
$$

where $F$ is an $M \times N$ matrix with $F_{i, j}=\frac{g\left(\mathbf{q}_{i}, \mathbf{r}_{j}\right) E_{\text {inc, }, \mathbf{p}_{i}}\left(\mathbf{r}_{j}\right) \Delta V}{E_{\text {inc, } \mathbf{p}_{i}}\left(\mathbf{q}_{i}\right)}$ and $O=\left[O\left(\mathbf{r}_{1}\right) O\left(\mathbf{r}_{2}\right) \cdots O\left(\mathbf{r}_{N}\right)\right]^{T}$. Using (13) for each measurement and stacking them together, we get

$$
\mathbf{P}_{\text {ryt }}=\operatorname{Imag}(\Phi),
$$

where $\mathbf{P}_{\mathrm{ryt}}=\frac{\mathbf{P}_{\mathrm{r}}(\mathrm{dBm})-\mathbf{P}_{\text {inc }}(\mathrm{dBm})}{10 \log _{10}\left(e^{-2}\right)}, \mathbf{P}_{\mathrm{r}}(\mathrm{dBm})=\left[P_{\mathrm{r}, \mathbf{p}_{1}}\left(\mathbf{q}_{1}\right)(\mathrm{dBm}) P_{\mathrm{r}, \mathbf{p}_{2}}\left(\mathbf{q}_{2}\right)(\mathrm{dBm}) \cdots P_{\mathrm{r}, \mathbf{p}_{M}}\left(\mathbf{q}_{M}\right)(\mathrm{dBm})\right]^{T}$, $\mathbf{P}_{\text {inc }}(\mathrm{dBm})=\left[P_{\mathrm{inc}, \mathbf{p}_{1}}\left(\mathbf{q}_{1}\right)(\mathrm{dBm}) P_{\mathrm{inc}, \mathbf{p}_{2}}\left(\mathbf{q}_{2}\right)(\mathrm{dBm}) \cdots P_{\mathrm{inc}, \mathbf{p}_{M}}\left(\mathbf{q}_{M}\right)(\mathrm{dBm})\right]^{T}$, and $P_{\mathrm{r}, \mathbf{p}_{i}}\left(\mathbf{q}_{i}\right)(\mathrm{dBm})$ 
and $P_{\text {inc, } \mathbf{p}_{i}}\left(\mathbf{q}_{i}\right)(\mathrm{dBm})$ are the received power and incident power corresponding to the transmitter and receiver pair $\left(\mathbf{p}_{i}, \mathbf{q}_{i}\right)$, respectively. Using (18) and (19), we get

$$
\mathbf{P}_{\mathrm{ryt}}=\operatorname{Real}(F O)=F_{\mathrm{R}} O_{\mathrm{R}}+F_{\mathrm{I}} O_{\mathrm{I}}
$$

where $\operatorname{Real}($.$) is the real part of the argument, and F_{\mathrm{R}}, F_{\mathrm{I}}, O_{\mathrm{R}}$ and $O_{\mathrm{I}}$ are the real part of $F$, imaginary part of $F$, real part of $O$, and imaginary part of $O$, respectively. This can be further simplified by noting that $F_{\mathrm{R}} O_{\mathrm{R}} \gg F_{\mathrm{I}} O_{\mathrm{I}}$ [29]. Therefore, the above equation becomes

$$
\mathbf{P}_{\mathrm{ryt}} \approx F_{\mathrm{R}} O_{\mathrm{R}}
$$

which is what we shall use for our RF-based robotic imaging.

\section{Intensity-Only LOS Approximation}

Starting from (6) and following similar steps to the intensity-only Rytov approximation, we get:

$$
P_{\mathrm{r}}(\mathbf{r})(\mathrm{dBm})=P_{\mathrm{inc}}(\mathbf{r})(\mathrm{dBm})-10 \log _{10}\left(e^{-2}\right) \omega \int_{\mathbb{L}_{T \rightarrow R}} \operatorname{Imag}\left(\alpha\left(\mathbf{r}^{\prime}\right)\right) d \mathbf{r}^{\prime},
$$

where the integration is the line integral along the line joining the positions of the transmitter and receiver, and $\mathbf{r}$ is the position of the receiver. Denoting $\mathbf{P}_{\mathrm{LOS}}=\frac{\mathbf{P}_{\mathrm{r}}(\mathrm{dBm})-\mathbf{P}_{\text {inc }}(\mathrm{dBm})}{10 \log _{10}\left(e^{-2}\right)}$ and stacking $M$ measurements together, we have

$$
\mathbf{P}_{\mathrm{LOS}}=A \Gamma,
$$

where $A$ is a matrix of size $M \times N$ with its entry $A_{i, j}=1$ if the $j^{\text {th }}$ cell is along the line joining the transmitter and receiver of the $i^{\text {th }}$ measurement, and $A_{i, j}=0$ otherwise, $\Gamma=$ $\left[\alpha_{\mathbf{I}}\left(\mathbf{r}_{1}\right) \alpha_{\mathbf{I}}\left(\mathbf{r}_{2}\right) \cdots \alpha_{\mathbf{I}}\left(\mathbf{r}_{N}\right)\right]^{T}$, and $\alpha_{\mathbf{I}}()=.\operatorname{Imag}(\alpha()$.$) .$

Equation (23) is what we then utilize in our setup when showing the performance of the state of the art.

\section{Brief Overview of Sparse Signal Processing [30]}

In the formulations of the Rytov and LOS approximations in Section II, we have a system of linear equations to solve for each approach. However, the system is severely underdetermined as the number of wireless measurements typically amount to a small percentage of the number of unknowns. More specifically, let $\mathbf{x} \in \mathbb{R}^{N}$ be a general unknown signal, $\mathbf{y} \in \mathbb{R}^{M}$ be the 
measurement vector, and $\mathbf{y}=B \mathbf{x}$ be the observation model, where $B$ is an $M \times N$ observation matrix. We consider the case where $N \gg M$, i.e., the number of unknowns is much larger than the number of measurements. Thus, it is a severely underdetermined problem which cannot be solved uniquely for $\mathbf{x}$ given $\mathbf{y}$. In this section, we briefly summarize how sparse signal processing can be utilized to solve this problem.

Suppose $\mathbf{x}$ can be represented as a sparse vector in another domain as follows: $\mathbf{x}=\Theta \mathbf{X}$, where $\Theta$ is an invertible matrix and $\mathbf{X}$ is $S$-sparse, i.e., card $(\operatorname{supp}(\mathbf{X})) \ll N$, where card $($.$) denotes the$ cardinality of the argument and $\operatorname{supp}($.$) denotes the set of indices of non-zero elements of the$ argument. Then, we have $\mathbf{y}=K \mathbf{X}$, where $K=B \Theta$ and $\mathbf{X}$ has a much smaller number of the non-zero elements than $\mathbf{x}$. In general, the solution to the above problem is obtained by solving the following non-convex combinatorial problem:

$$
\text { minimize }\|\mathbf{X}\|_{0}, \quad \text { subject to } \quad \mathbf{y}=K \mathbf{X} \text {. }
$$

Since solving (24) is computationally-intensive and impractical, considerable research has been devoted towards developing approximated solutions for (24).

In our case, we are interested in imaging and localization of the objects in an area. Spatial variations of the objects in a given area are typically sparse. We thus take advantage of the sparsity of the spatial variations to solve our under-determined system. ${ }^{7}$ More specifically, let $R=\left[R_{i, j}\right]$ denote an $m \times n$ matrix that represents the unknown space. Since we are interested in the spatial variations of $R$, let

$$
D_{h, i, j}=\left\{\begin{array}{ll}
R_{i+1, j}-R_{i, j} & \text { if } 1 \leqslant i<m, \\
R_{i, j}-R_{1, j} & \text { if } i=m,
\end{array} \text { and } D_{v, i, j}= \begin{cases}R_{i, j+1}-R_{i, j} & \text { if } 1 \leqslant j<n \\
R_{i, j}-R_{i, 1} & \text { if } j=n .\end{cases}\right.
$$

Then, the Total Variation (TV) of $R$ is defined as:

$$
\operatorname{TV}(R)=\sum_{i, j}\left\|\mathbf{D}_{i, j}(R)\right\|
$$

where $\mathrm{D}_{i, j}(R)=\left[D_{h, i, j} D_{v, i, j}\right]$, and $\|$.$\| can represent either l_{1}$ or $l_{2}$ norm. TV minimization then solves the following convex optimization problem:

$$
\text { minimize } \operatorname{TV}(R), \quad \text { subject to } \mathbf{y}=K \mathbf{X} \text {. }
$$

\footnotetext{
${ }^{7}$ It is also possible to solve an $l_{1}$ convex relaxation of (24). However, our past analysis has indicated a better performance with spatial variation minimization [20].
} 
In the context of the current problem formulation, $\mathbf{X}$ represents the object map $\mathbf{D}, R$ represents the spatial variations of $\mathbf{X}, K$ represents the observation model, i.e., $K=F_{R}$ for the Rytov approach and $K=A$ for the LOS approach, and y represents the received power (after removing path loss). In solving (26), $l_{1}$ or $l_{2}$ norm results in a similar reconstruction [31]. Thus, unless otherwise stated, all results of this paper are based on $l_{1}$ norm.

To solve the general compressive sensing problem of (26) robustly and efficiently, TVAL3 (TV Minimization by Augmented Lagrangian and Alternating Direction Algorithms) is proposed in [32]. TVAL3 is a MATLAB-based solver that solves (26) by minimizing the augmented Lagrangian function using an alternating minimization scheme [33]. The augmented Lagrangian function includes coefficients which determine the relative importance of the terms $\operatorname{TV}(R)$ and $\|\mathbf{y}-K \mathbf{X}\|$ in (26). The readers are referred to [32] for more details on TVAL3. We use TVAL3 for all the experimental results of this paper.

\section{EXPERIMENT SETUP}

In this section, we briefly describe our enabling experimental testbed. As compared to our past testbed (results of which with an LOS modeling of the receptions were reported in the literature), in our current setup, the robots can take channel measurements over a given route autonomously, and without any coordination between themselves or stopping. More specifically, in [24], the two robots had to constantly stop and coordinate their positioning and antenna alignment, and their positioning errors were manually corrected a few times in a route (e.g. every $1 \mathrm{~m}$ ). In this paper, each robot travels a route autonomously and without any coordination with the other robot or positioning error correction. It is therefore feasible to collect measurements much faster, reducing the experiment time from several hours to a few minutes. However, this comes at the cost of non-negligible errors in robot positioning and antenna alignment, as we discuss later in the paper. In the rest of this section, we describe the software and hardware aspects of our testbed in more details, emphasizing the main differences from our previously-reported testbed [34].

In our setup, we use two Pioneer 3-AT (P3-AT) mobile robots from MobileRobots Inc. [35], each equipped with an onboard PC, and an IEEE 802.11g (WLAN) card. Each robot can simultaneously follow a given path and take the corresponding received signal strength measurements (RSSI) as it moves. The data is then stored and transferred back to a laptop at 
the end of the operation.

\section{A. Hardware Architecture}

P3-AT mobile robots [35] are designed for indoor, outdoor, and rough-terrain implementations. They feature an onboard PC104 and a Renesas SH7144-based micro-controller platform for control of the motors, actuators and sensors. By utilizing a $\mathrm{C} / \mathrm{C}++$ application programming interface (API) library provided by MobileRobots, users are able to program and control the robot via the micro-controller platform. Fig. 2 shows the P3-AT robot. We have furthermore utilized directional antennas for better imaging results. In order to hold the directional antennas, we have built an additional electromechanical fixture, as can be seen from Fig. 2. This antenna is rotated and positioned via a Hitec HA-7955TG digital servo mounted on the antenna fixture. Via a serial port, PWM values are passed from the onboard PC104 to a Digilent Cerebot II micro-controller on the side of the antenna frame. These PWM waveforms are then outputted to the Hitec Servo, specifying a range of 0 - 180 degree angle. We use a GD24-15 2.4 GHz parabolic grid antenna from Laird Technologies [36]. This model has a $15 \mathrm{dBi}$ gain with 21 degree horizontal and 17 degree vertical beamwidth and is suitable for IEEE $802.11 \mathrm{~b} / \mathrm{g}$ applications.

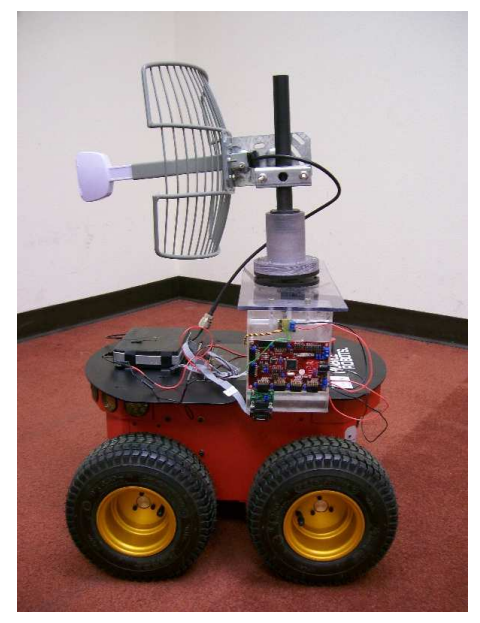

Fig. 2. The figure shows a Pioneer 3-AT robot with the additionally-mounted servomechanism and a directional antenna.

One of the robots has a D-Link WBR-1310 wireless router attached to its antenna. It constantly outputs a wireless signal for the other robot to measure the signal strength. The overall operation is overseen by a remote $\mathrm{PC}$, which is in charge of passing the initial plan to the robots to execute, 
and collecting the final signal strength readings at the end of the operation. A block diagram of the hardware architecture of the robots is shown in Fig. 3, and is similar to what we have used in [34].

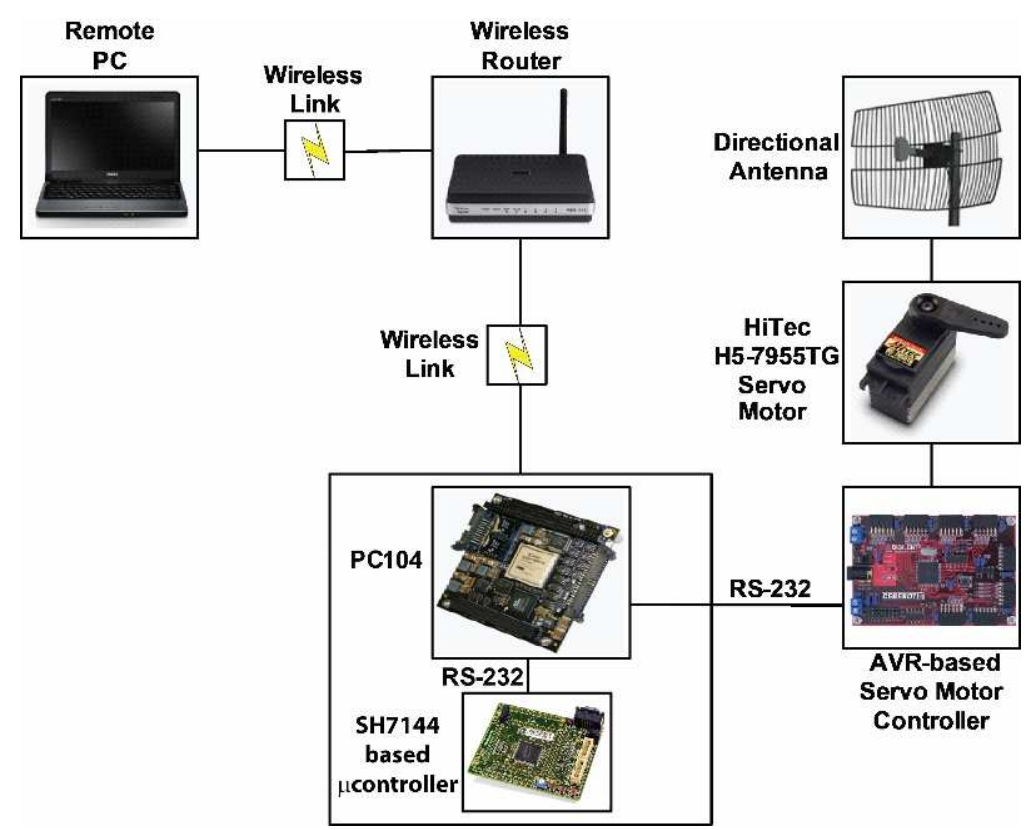

Fig. 3. Block diagram of the hardware architecture of one of the robots.

\section{B. Software Architecture}

The overall software architecture of our system can be seen in Fig. 4. The software system is composed of two application layers, one running on a remote PC to control the experiment and one running on the robots themselves. The programs are developed in C++ using the ARIA library developed by MobileRobots. They communicate via a TCP/IP connection between the robot-side application, which acts as the server, and the PC-side application, which acts as the client. The remote PC is in charge of overseeing the whole operation and giving initial control commands and route information to the robots. The user can specify the route information involving the direction of movement, the length of the route and the coordinates of the starting positions of the robots. While the overall software structure is similar to our previous work [24], significant changes are made in programming each block of Fig. 4. These changes enable a different level of autonomy as compared to our previous work. Next, we explain these changes made in the software in more details. 
In order to synchronize all the operations - robot movement, antenna alignment and signal strength measurement, the robot execution is divided into four separate in-software threads: the antenna control thread, signal strength thread, motor control thread, and main thread, which respectively control the antenna rotation, manage the reading of the wireless signal strength, operate the motor such as in driving forward, and send the overall commands. The main thread initializes/finalizes other threads and communicates with the remote PC. Before a route begins, the main thread first creates the threads needed to run the other operations and freezes their operations using Mutex. It then receives the path information of both robots from the remote PC. This information is passed to the antenna control and signal strength threads, where it will be used to calculate when to read the signal strength, and how to rotate the antenna over the route to keep the antennas on both robots aligned. Once the threads are properly initialized, the path information is passed to the motor control thread to begin the operation. The measurements gathered by one robot will be stored on its PC and are transferred back to the remote PC at the end of the operation. This is because any kind of TCP communication introduces unnecessary delays in the code during the measurements. It is necessary, however, to be able to control the robot movement and operation at all times from the remote PC in case of emergency. Therefore, the code is designed to maximize the autonomy and precision of the operation, through threading, while being able to shut down via remote control at any time. This is achieved with the main thread utilizing a polling approach.

\section{Robot Positioning}

Accurate positioning is considerably important as the robots need to constantly put a position stamp on the locations where they collect the channel measurements and further align their antennas based on the position estimates. In our setup, our robots utilize on-board gyroscopes and wheel encoders to constantly estimate their positions. Since we use a dead reckoning approach to localize our robots, timing is very important to the accuracy of position estimation. We thus employ precise timers in software to help the robot determine its own position as well as the position of the other robot based on the given speed. More specifically, when the motor control thread begins its operation, timers are simultaneously initiated in all the threads, allowing them to keep track of when and where they are in their operations. Also, the threads' Mutex are released, allowing the robots to move and take measurements. 


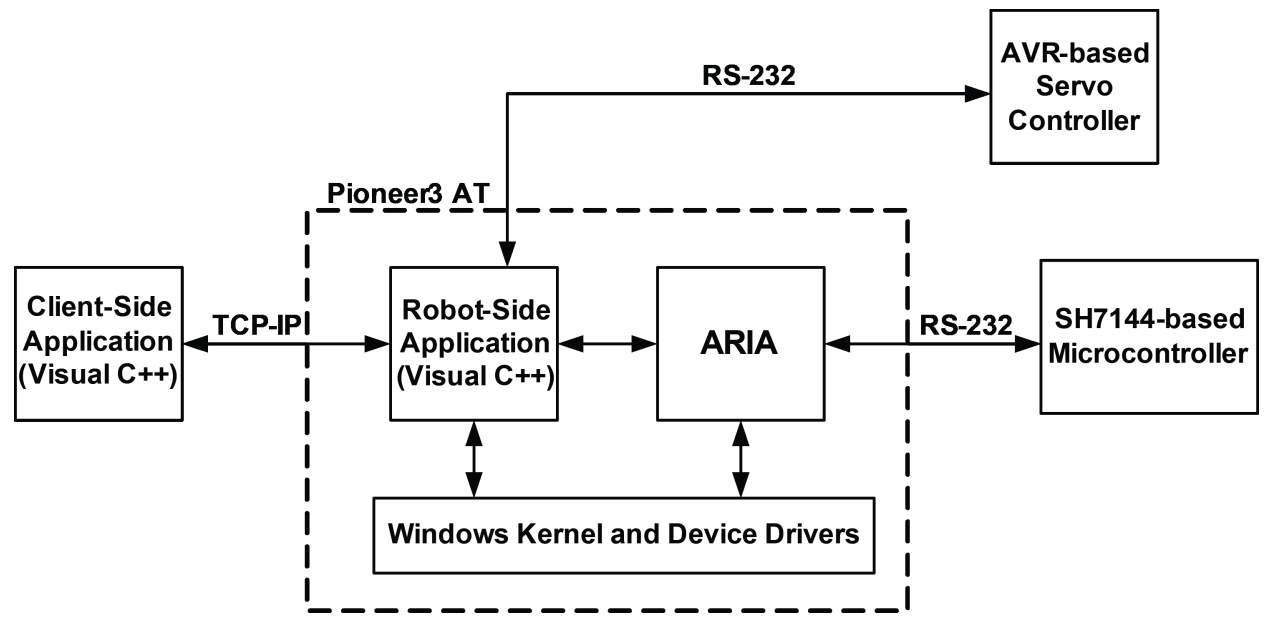

Fig. 4. Software architecture of the robot platform.

It is important to note that once the robots start a route, there is no communication or coordination between them. Each robot constantly uses the set speed and timer information to estimate its own location as well as the location of the other robot for antenna alignment and measurement collection. Thus, all the measurements and alignments are naturally prone to positioning errors. Currently, we use speeds up to $10 \mathrm{~cm} / \mathrm{s}$. A sample route is shown in Fig. 5 (see the 0 degree angle route, for instance). Our current localization error is less than $2.5 \mathrm{~cm}$ per one meter of straight line movement, and our current considered routes typically span 10 - 20 meters. Additionally, the robot also experiences a drift from the given path. These robot positioning errors will also result in antenna alignment errors. In Section V, we discuss the impact of both errors on our imaging results in details.

\section{Robot Paths}

So far, we have explained the hardware and software aspects of the experimental testbed. Next, we briefly explain the routes that the robots would take. More specifically, the transmitting and receiving robots move outside of $\mathbf{D}$, similar to how CT-scan is done, in parallel, along the lines that have an angle $\theta$ with the $x$-axis. This means that the line connecting the transmitter and receiver would ideally (in the absence of positioning errors) stay orthogonal to the line with angle $\theta$. Sample routes along 0 and 45 degree angles are shown in Fig. 5. Both of the robots move with a same velocity of $10 \mathrm{~cm} / \mathrm{s}$ and take measurements every $0.2 \mathrm{sec}$ (i.e., measurements 
are taken with $2 \mathrm{~cm}$ resolution). As explained earlier, there is no coordination between the robots when traveling a route. To speed up the operation, we currently manually move the robots from the end of one route to the beginning of another route. This part can also be automated as part of future work. Additionally, random wireless measurements, a term we introduced in [24], where the transmitter is stationary and the receiver moves along a given line, can also be used. In the next section, we only consider the case of parallel routes, as shown in Fig. 5. Readers are referred to [34] for more details and tradeoff analysis on using parallel or random routes in the context of LOS reception modeling.

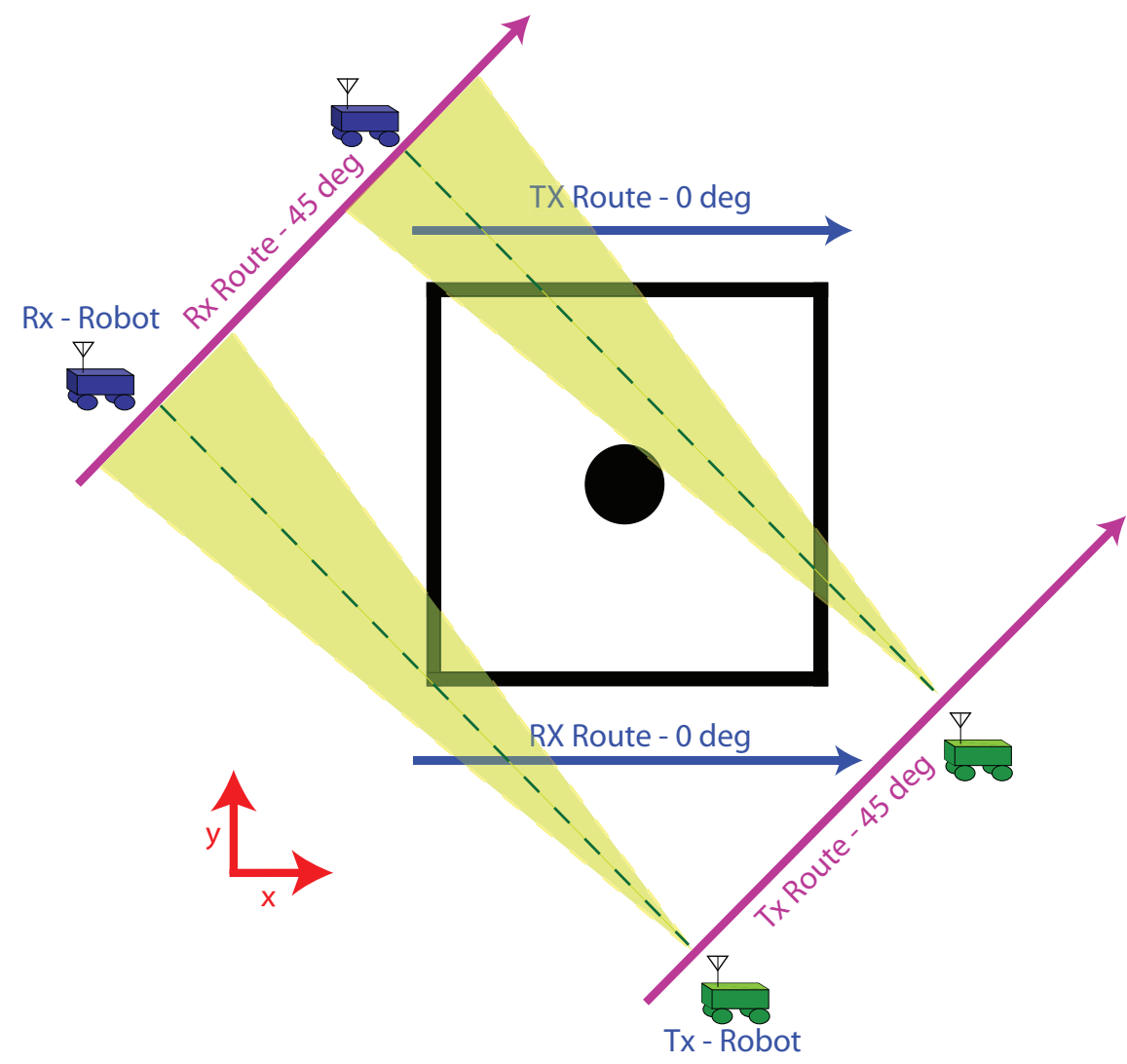

Fig. 5. Sample routes for measurement collection are shown for 0 and 45 degree angles.

\section{EXPERIMENTAL RESUlts AND Discussions}

In this section, we show the results of see-through wall imaging with Rytov wave approximation and further compare them with the state of the art results based on LOS modeling. We consider three different areas, as shown in Fig. 8, 9 and 10 (top-left). We name these cases as 
follows for the purpose of referencing in the rest of the paper: T-shape, occluded cylinder, and occluded two columns. Two robots move outside of the area of interest and record the signal strength. These measurements are then used to image the corresponding unknown regions using both Rytov and LOS approaches, as described in Section II. Fig. 8, 9 and 10 further show the horizontal cuts of these areas. In this paper, we only consider 2D imaging, i.e., imaging a horizontal cut of the structure.

Fig. 6 shows a sample of the real measurement along the 0 degree line for the T-shape, with the distance-dependent path loss component removed. As mentioned previously, the distancedependent path loss component does not contain any information about the objects. Thus, by making a few measurements in the same environment where there are no objects between the robots, it is estimated and removed. To compare how well the WKB (LOS modeling) and Rytov approximations match the real measurements, the simulated received signal loss using each approximation is plotted in Fig. 7 for the route along the 0 degree angle for the occluded cylinder structure. As can be seen, the Rytov approximation matches the real measurement considerably better than the LOS modeling.

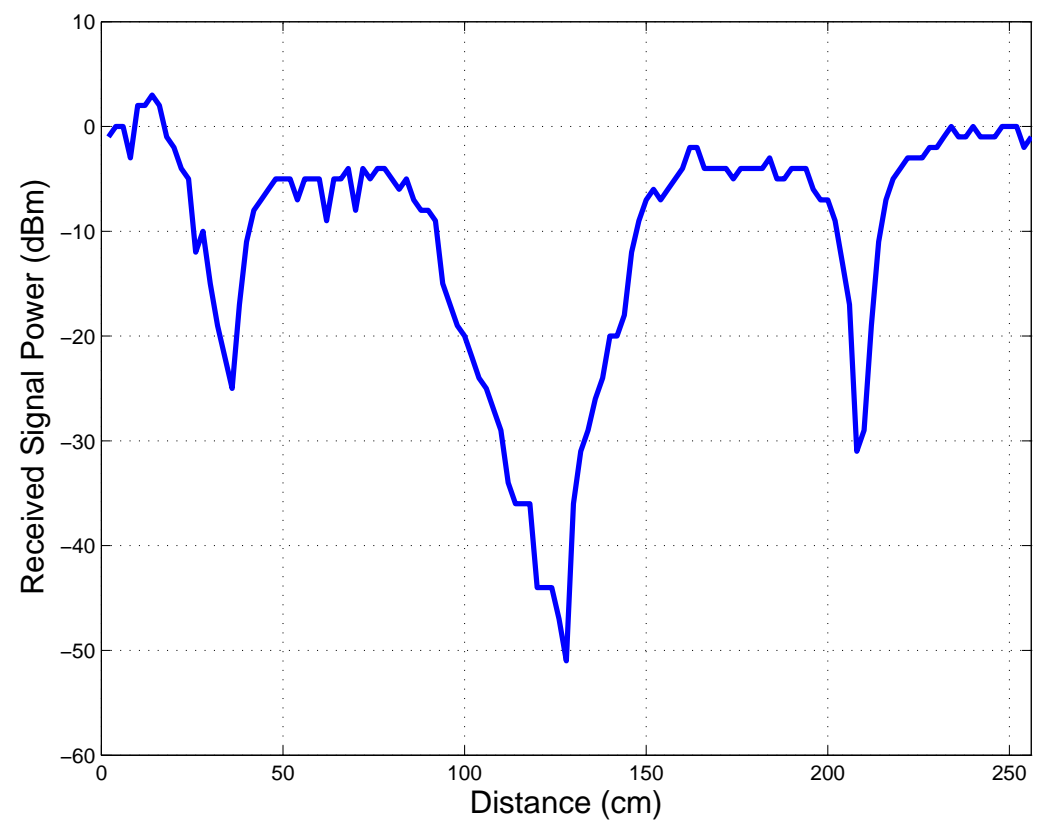

Fig. 6. Real received signal power along the 0 degree line for the T-shape, with the distance-dependent path loss component removed. 


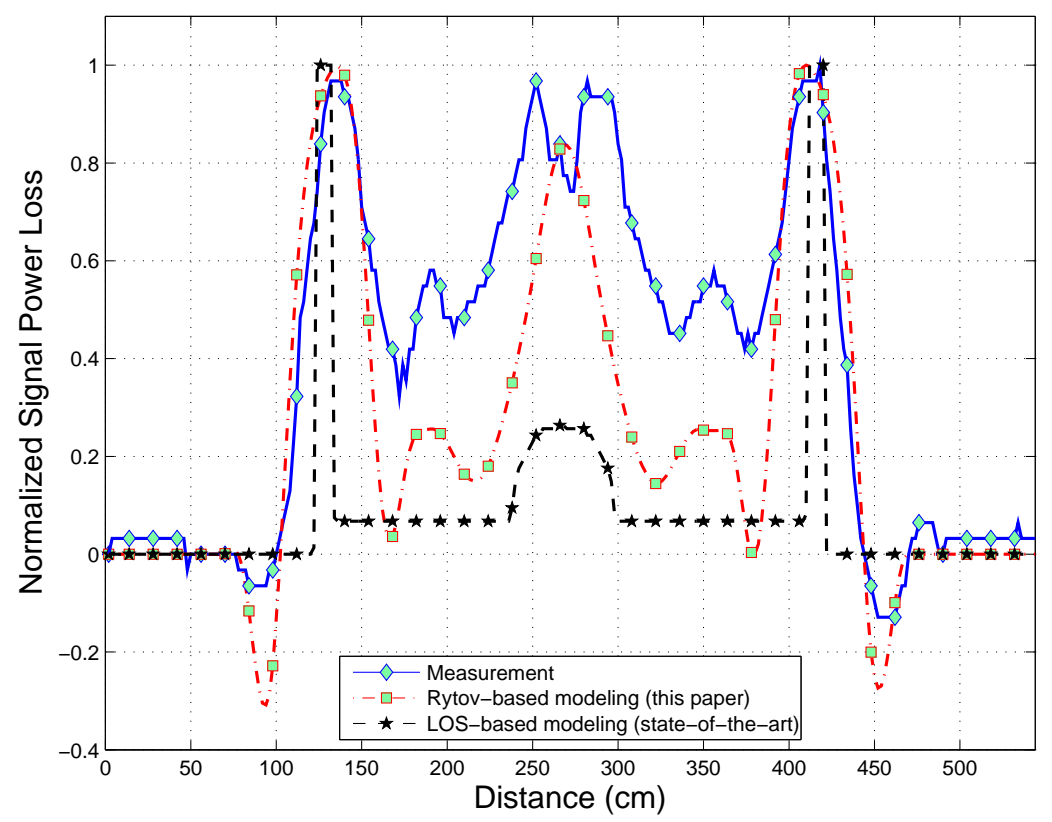

Fig. 7. Comparisons of the Rytov and LOS approximations for the route along the 0 degree angle for the occluded cylinder. As can be seen, the Rytov approximation matches the real measurement considerably better than the LOS modeling through WKB approximation.

Our imaging results for the T-shape, the occluded cylinder and the occluded two columns are shown in Fig. 8, 9 and 10 respectively. For the T-shape and the occluded cylinder, we have measurements along four angles of 0,90, 45, and 135 degrees. For the occluded two columns we have measurements along five angles of 0,90, 80,-10 and 10 degrees. The total measurements thus amount to only $20.12 \%, 4.7 \%$ and $2.6 \%$ for the T-shape, the occluded cylinder, and the occluded two columns respectively. Fig. 8 (left) shows the T-shape structure with its horizontal cut marked. This horizontal cut, which is the true original image that the robots need to construct, is an area of $0.64 \mathrm{~m} \times 1.82 \mathrm{~m}$, which results in 2912 unknowns to be estimated. Fig. 8 further shows the imaging results with both Rytov and LOS for this structure. As can be seen, Rytov provides a considerably better imaging quality, especially around the edges. The reconstructions after thresholding are also shown in Fig. 8, which uses the fact that we are interested in a black/white image that indicates absence/presence of objects (more details on this will follow soon).

Fig. 9 shows the imaging of the occluded cylinder. This area of interest is $2.98 \mathrm{~m} \times 2.98 \mathrm{~m}$, 
amounting to 22201 unknowns to be estimated based on only $4.7 \%$ measurements. This structure is more challenging than the T-shape to reconstruct because 1) it is fully behind thick brick walls, and 2) it consists of materials with different properties (metal and brick). Similarly, we can see that Rytov provides a better imaging result for this structure as well, with the details reconstructed more accurately. Thresholded images are also shown.

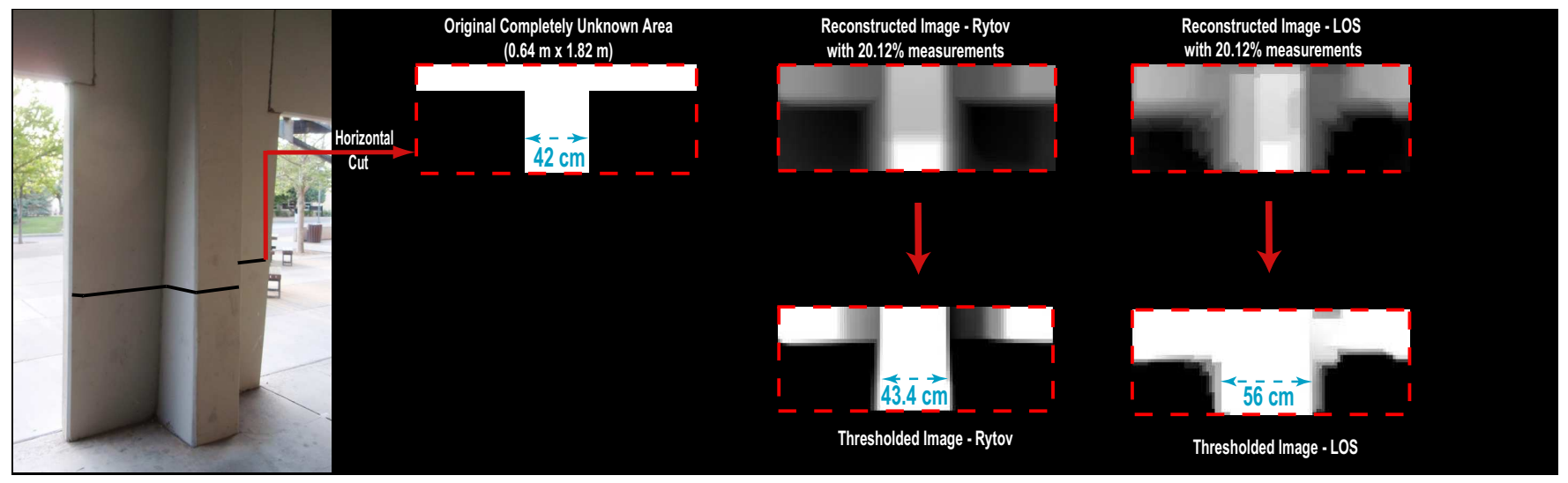

Fig. 8. The left figures show the T-shape structure of interest that is completely unknown and needs to be imaged, as well as its horizontal cut (its dimension is $0.64 \mathrm{~m} \times 1.82 \mathrm{~m}$ ). The white areas in the true image indicate that there is an object while the black areas denote that there is nothing in those spots. Imaging results based on $20.12 \%$ measurements are shown for both Rytov and LOS approaches. Sample dimensions of the original and the reconstructed images are also shown. It can be seen that Rytov provides a considerably better imaging result.

Fig. 10 shows the imaging of the occluded two columns. This area of interest is $4.56 \mathrm{~m} \times$ $5.74 \mathrm{~m}$ (amounting to 65436 unknowns) and is estimated only with $2.6 \% \mathrm{WiFi}$ measurements. This structure is more challenging to image than both the T-shape and the occluded cylinder since 1) there are two columns close to each other, which results in a higher multipath and other propagation phenomena and, 2) smaller percentage of measurements are available for imaging (half of that used for the occluded cylinder). The figure shows the thresholded imaging results as well. More specifically, any value above $40 \%$ and below $20 \%$ of the maximum value is thresholded to the $40 \%$ and $20 \%$ values respectively (the same thresholding approach is used for the past two areas). As can be seen from Fig. 10, the LOS approach fails to image this more complex structure while Rytov can image it. From Fig. 8 and 9, it can be seen that imaging based on LOS modeling can vaguely image the details. But for more complex areas such as Fig. 10 , its performance becomes unacceptable while Rytov can locate the objects fairly accurately. 


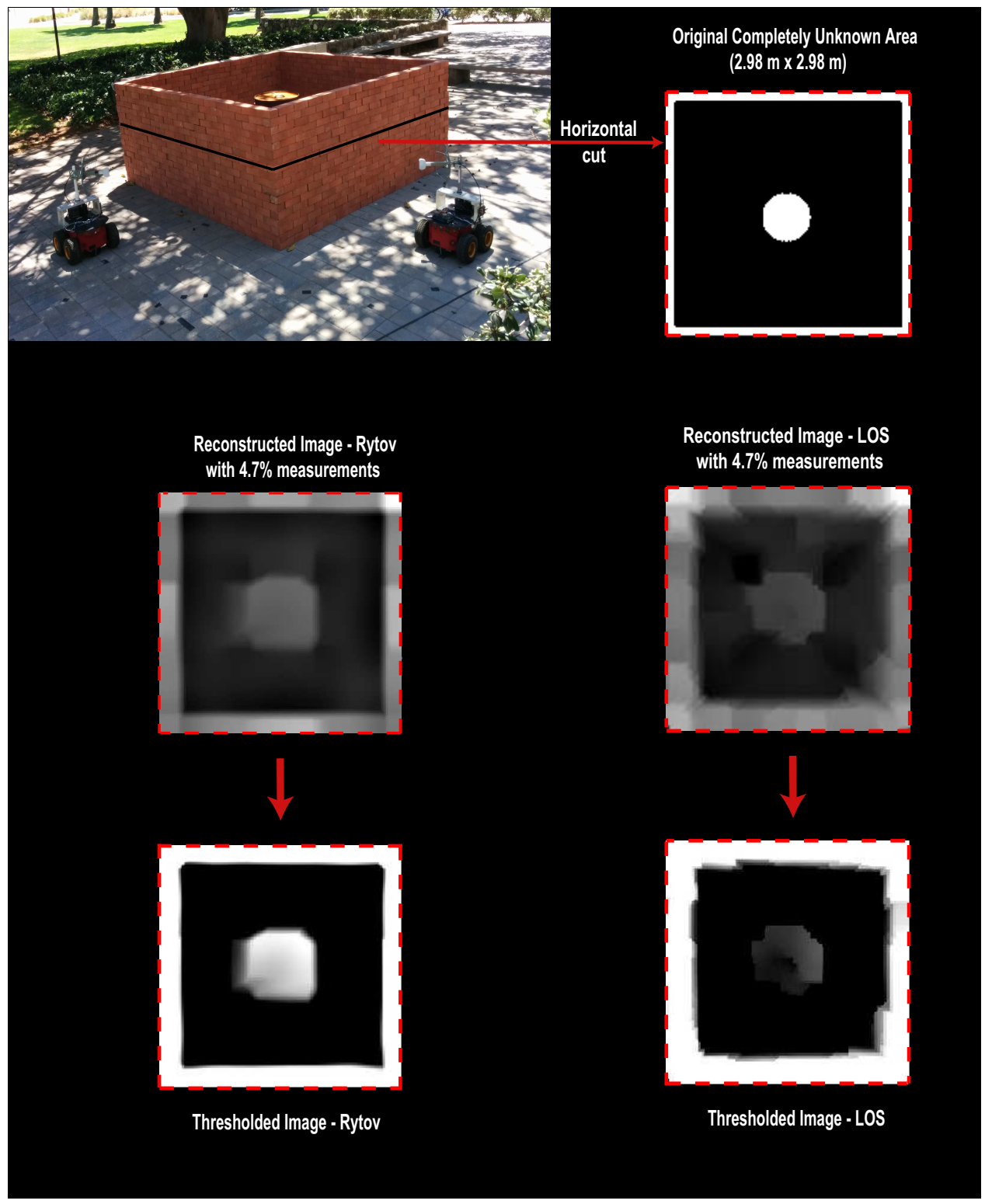

Fig. 9. The left figures show the occluded cylinder structure of interest that is completely unknown and needs to be imaged, as well as its horizontal cut (its dimension is $2.98 \mathrm{~m} \times 2.98 \mathrm{~m}$ ). The white areas in the true image indicate that there is an object while the black areas denote that there is nothing in those spots. Imaging results based on $4.7 \%$ measurements are shown for both Rytov and LOS approaches. It can be seen that Rytov provides a considerably better imaging result.

This signifies the importance of properly modeling the receptions.

In general, the computational complexity of our imaging approach depends on the size of the unknown area and the number of gathered measurements. Furthermore, the utilized solver typically converges faster if the model better matches the real measurements. Hence, we expect 


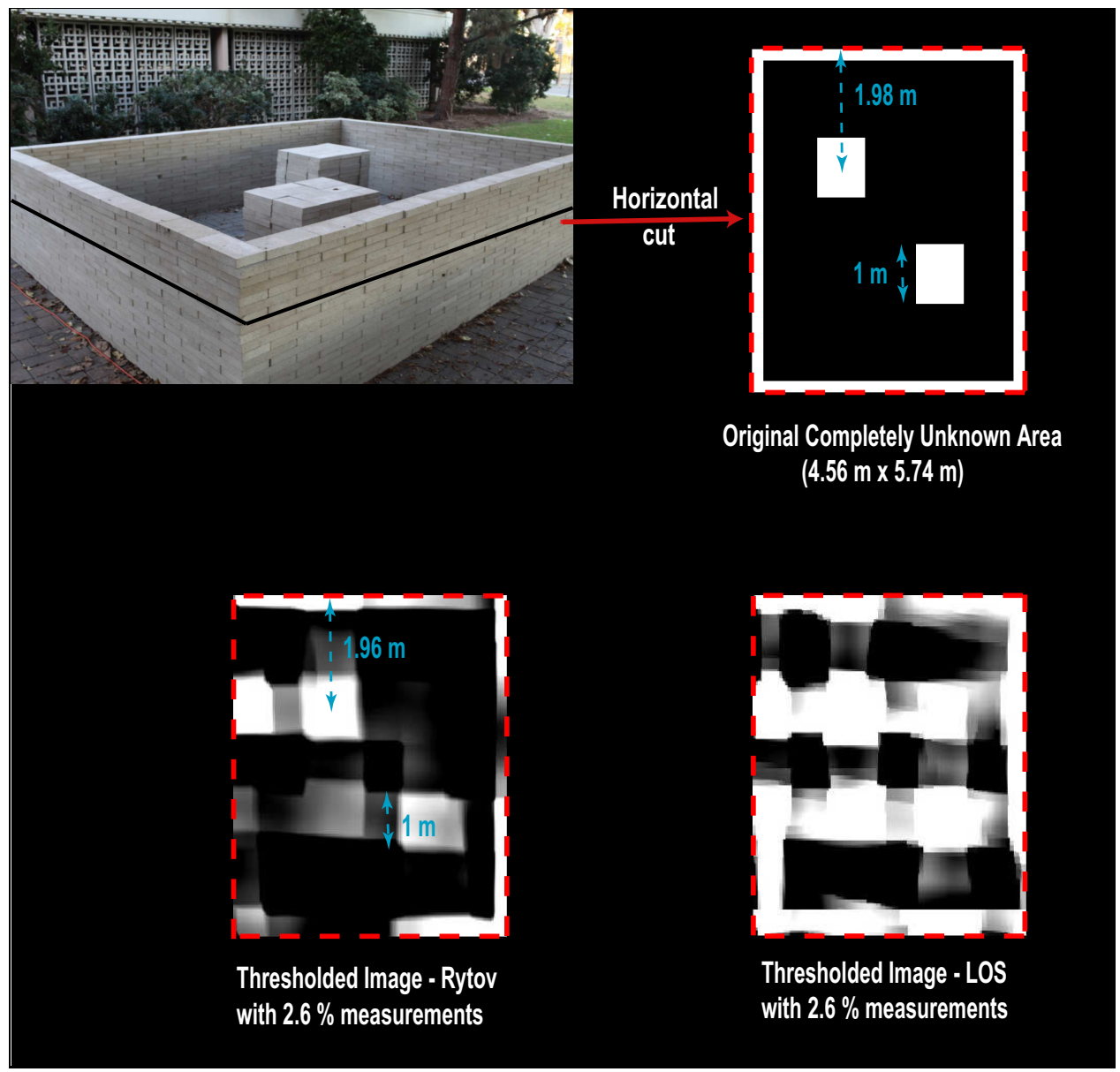

Fig. 10. The top figures show the occluded two columns structure of interest that is completely unknown and needs to be imaged, as well as its horizontal cut (its dimension is $4.56 \mathrm{~m} \times 5.74 \mathrm{~m}$ ). The white areas in the true image indicate that there is an object while the black areas denote that there is nothing in those spots. Imaging results based on $2.6 \%$ measurements are shown in the bottom figures for both Rytov and LOS approaches. Sample dimensions are also shown. It can be seen that the LOS approach fails to properly image the occluded objects while Rytov performs significantly better.

that the Rytov approach runs faster than LOS approach because of its better match with the real measurement. We verify this on a desktop equipped with a $3.7 \mathrm{GHz} \mathrm{CPU}$. For the T-shape with 4096 unknowns and 586 measurements, the Rytov approach takes 3.6 seconds, while the LOS approach takes 5.74 seconds. For the occluded cylinder with 22201 unknowns and 1036 measurements, the Rytov approach takes 17.01 seconds, while the LOS approach takes 27.14 seconds. For the occluded two columns inside with 65436 unknowns and 1699 measurements, the Rytov approach takes 54.8 seconds, while the LOS approach takes 64 seconds. However, it should be noted that Rytov also requires an offline calculation of the $F_{\mathrm{R}}$ matrix for a given set 
of routes. This takes 9 minutes for the occluded cylinder structure for example. Once this matrix is calculated, it can be used for any setup that uses the same routes.

Finally, we note that the measurements of the T-shape were collected with our past experimental setup since we do not have access to this site anymore. However, we expect similar results with our new experimental setup for this site, for the reasons explained in Section V-A.

\section{A. Effect of robot positioning and antenna alignment errors}

As each robot travels a route autonomously and without coordination with the other robot or positioning error correction, there will be non-negligible positioning and antenna alignment errors accumulated throughout the route. We next show the impact of these errors on our see-through imaging performance.

Fig. 11 and 12 show the impact of localization and antenna alignment errors on Rytov and LOS approaches respectively. More specifically, each figure compares experimental imaging results of three cases with different levels of localization/antenna alignment errors. The most accurate localization case was generated with our old setup where positioning errors were corrected every $1 \mathrm{~m}$. The middle and right cases are both automated but the robot has different speeds, which results in different positioning accuracy. In each case, the positioning error leads to a non-negligible antenna alignment error, the value of which is reported (as a \% of the antenna beamwidth). However, we can see that the combination of both antenna alignment and positioning errors, which are not negligible, has a negligible impact on the imaging result. This is due to the fact that the main current bottleneck in see-through imaging is the modeling of the receptions, which is the main motivation for this paper. For instance, as we showed in Fig. 7, the gap between the state of the art modeling (LOS) and the true receptions is huge, which we have reduced considerably by a proper modeling of the receptions in this paper. However, the gap is still non-negligible as compared to other sources of errors such as robot positioning and antenna alignment errors, as Fig. 11 and 12 confirm. It is needless to say that if these errors become more considerable, they will inevitably start impacting the results. Thus, Fig. 11 and 12 imply that with our current setup and the size of the areas we imaged in this paper, the impact of robot positioning and antenna alignment errors was negligible on our results. 


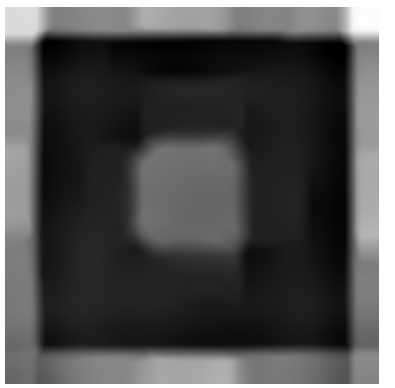

(a) Length of the Route $=4.88 \mathrm{~m}$

Accumulated robot positioning error of the route $=2 \mathrm{~cm}$

Antenna alignment error $=0 \%$

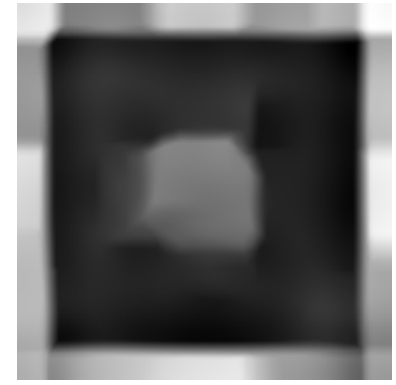

(b) Length of the Route $=4.88 \mathrm{~m}$

Accumulated robot positioning error of the route $=18.7 \mathrm{~cm}$

Antenna alignment error $=9.5 \%$

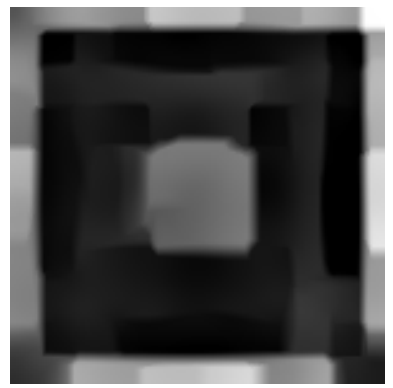

(c) Length of the Route $=4.88 \mathrm{~m}$

Accumulated robot positioning error of the route $=22.36 \mathrm{~cm}$

Antenna alignment error $=27 \%$

Fig. 11. The figure shows the effect of robot positioning and antenna alignment errors on imaging based on Rytov approximation. It can be seen that they have negligible impact.

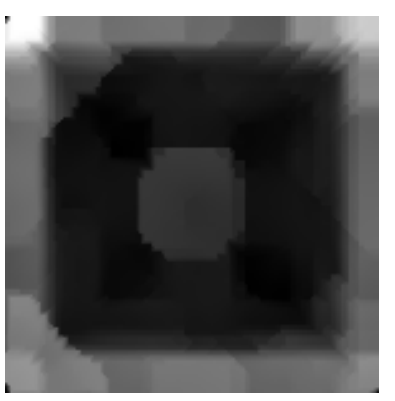

(a) Length of the Route $=4.88 \mathrm{~m}$

\section{Accumulated robot positioning error of the route $=2 \mathrm{~cm}$}

Antenna alignment error $=0 \%$

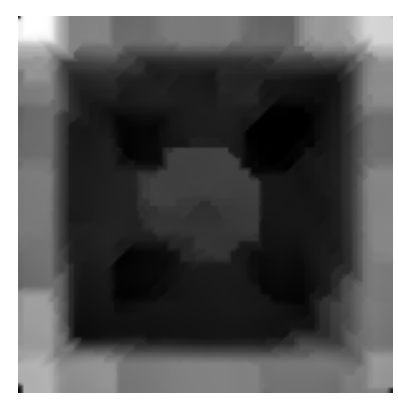

(b) Length of the Route $=4.88 \mathrm{~m}$

Accumulated robot positioning error of the route $=18.7 \mathrm{~cm}$

Antenna alignment error $=9.5 \%$

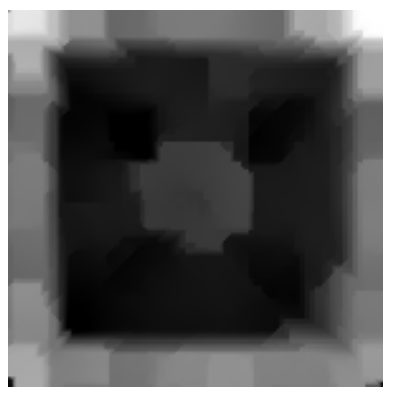

(c) Length of the Route $=4.88 \mathrm{~m}$

Accumulated robot positioning error of the route $=22.36 \mathrm{~cm}$

Antenna alignment error $=27 \%$

Fig. 12. The figure shows the effect of robot positioning and antenna alignment errors on imaging based on LOS modeling. It can be seen that they have negligible impact.

\section{CONCLUSiOnS AND Future WorK}

In this paper, we have considered the problem of high-resolution imaging through walls, with only WiFi signals, and its automation with unmanned vehicles. We have developed a theoretical 
framework for this problem based on Rytov wave models, sparse signal processing, and robotic path planning. We have furthermore validated the proposed approach on our experimental robotic testbed. More specifically, our experimental results have shown high-resolution imaging of three different areas based on only a small number of WiFi measurements (20.12\%, 4.7\% and 2.6\%). Moreover, they showed considerable performance improvement over the state-of-the-art that only considers the Line Of Sight path, allowing us to image more complex areas not possible before. Finally, we showed the impact of robot positioning and antenna alignment errors on our see-through imaging framework. Overall, the paper addresses one of the main bottlenecks of see-through imaging, which is the proper modeling of the receptions. Further improvement to the modeling, while maintaining a similar computational complexity, is among future directions of this work.

\section{ACKNOWLEDGEMENTS}

The authors would like to acknowledge the help of Yuan Yan with proofreading the paper. Furthermore, they would like to thank Herbert Cai and Zhengli Zhao for their help with running the experiments.

\section{REFERENCES}

[1] X. Chen, A. Edelstein, Y. Li, M. Coates, M. Rabbat, and A. Men. Sequential monte carlo for simultaneous passive devicefree tracking and sensor localization using received signal strength measurements. In Proceedings of the 10th International Conference on Information Processing in Sensor Networks (IPSN), pages 342-353, 2011.

[2] Y. Chen, D. Lymberopoulos, J. Liu, and B. Priyantha. Fm-based indoor localization. In Proceedings of the 10th international conference on Mobile systems, applications, and services, pages 169-182, 2012.

[3] A. Kosba, A. Saeed, and M. Youssef. Rasid: A robust WLAN device-free passive motion detection system. In IEEE International Conference on Pervasive Computing and Communications (PerCom), pages 180-189, 2012.

[4] M. Moussa and M. Youssef. Smart cevices for smart environments: Device-free passive detection in real environments. In IEEE International Conference on Pervasive Computing and Communications (PerCom), pages 1-6, 2009.

[5] R. Nandakumar, K. Chintalapudi, and V. Padmanabhan. Centaur: locating devices in an office environment. In Proceedings of the 18th annual international conference on Mobile computing and networking, pages 281-292, 2012.

[6] H. Schmitzberger and W. Narzt. Leveraging wlan infrastructure for large-scale indoor tracking. In Proceedings of the 6th International Conference on Wireless and Mobile Communications (ICWMC), pages 250-255, 2010.

[7] M. Bocca, S. Gupta, O. Kaltiokallio, B. Mager, Q. Tate, S. Kasera, N. Patwari, and S. Venkatasubramanian. RF-based device-free localization and tracking for ambient assisted living.

[8] T. Bailey and H. Durrant-Whyte. Simultaneous localization and mapping (slam): Part I the essential algorithms. IEEE Robotics and Automation Magazine, 13(2):99 - 110, 2006. 
[9] T. Bailey and H. Durrant-Whyte. Simultaneous localization and mapping (slam): Part II. IEEE Robotics \& Automation Magazine, 13(3):108-117, 2006.

[10] S. Thrun, W. Burgard, and D. Fox. A probabilistic approach to concurrent mapping and localization for mobile robots. Autonomous Robots, 31(1-3):29-53, 1998.

[11] F. Dellaert, F. Alegre, and E. Martinson. Intrinsic localization and mapping with 2 applications: Diffusion mapping and macro polo localization. In International Conference on Robotics and Automation, pages 2344-2349, 2003.

[12] Ebi Jose and Martin David Adams. An augmented state slam formulation for multiple line-of-sight features with millimetre wave radar. In Intelligent Robots and Systems, 2005.(IROS 2005). 2005 IEEE/RSJ International Conference on, pages 3087-3092. IEEE, 2005.

[13] W. Chew. Waves and fields in inhomogeneous media, volume 522. IEEE press New York, 1995.

[14] W. Chew and Y. Wang. Reconstruction of two-dimensional permittivity distribution using the distorted born iterative method. IEEE Transactions on Medical Imaging, 9(2):218-225, 1990.

[15] L.-P. Song, C. Yu, and Q. Liu. Through-wall imaging (twi) by radar: 2-D tomographic results and analyses. IEEE Transactions on Geoscience and Remote Sensing, 43(12):2793-2798, 2005.

[16] Q. Liu, Z. Zhang, T. Wang, J. Bryan, G. Ybarra, L. Nolte, and W. Joines. Active microwave imaging. I. 2-D forward and inverse scattering methods. IEEE Transactions on Microwave Theory and Techniques, 50(1):123-133, 2002.

[17] Y.-H. Chen and M. Oristaglio. A modeling study of borehole radar for oil-field applications. Geophysics, 67(5):1486-1494, 2002.

[18] P. Van Den Berg, A. Van Broekhoven, and A. Abubakar. Extended contrast source inversion. Inverse Problems, 15(5):1325, 1999.

[19] M. Pastorino. Stochastic optimization methods applied to microwave imaging: A review. IEEE Transactions on Antennas and Propagation, 55(3):538-548, 2007.

[20] Y. Mostofi. Cooperative wireless-based obstacle/object mapping and see-through capabilities in robotic networks. IEEE Transactions on Mobile Computing, 12(5):817-829, 2013.

[21] Y. Mostofi and P. Sen. Compressive Cooperative Mapping in Mobile Networks. In Proceedings of the 28th American Control Conference (ACC), pages 3397-3404, St. Louis, MO, June 2009.

[22] G. Oliveri, L. Poli, P. Rocca, and A. Massa. Bayesian compressive optical imaging within the Rytov approximation. Optics Letters, 37(10):1760-1762, 2012.

[23] G. Tsihrintzis and A. Devaney. Higher order (nonlinear) diffraction tomography: Inversion of the Rytov series. IEEE Transactions on Information Theory, 46(5):1748-1761, 2000.

[24] Y. Mostofi. Compressive cooperative sensing and mapping in mobile networks. IEEE Transactions on Mobile Computing, 10(12):1769-1784, 2011.

[25] L. Tsang, J. Kong, and K.-H. Ding. Scattering of Electromagnetic Waves, Theories and Applications, volume 27. John Wiley \& Sons, 2004.

[26] J. Shea, P. Kosmas, S. Hagness, and B. Van Veen. Three-dimensional microwave imaging of realistic numerical breast phantoms via a multiple-frequency inverse scattering technique. Medical physics, 37(8):4210-4226, 2010.

[27] K. Avinash and M. Slaney. Principles of computerized tomographic imaging. Society for Industrial and Applied Mathematics, 2001.

[28] A. Devaney. A filtered backpropagation algorithm for diffraction tomography. Ultrasonic imaging, 4(4):336-350, 1982. 
[29] A. Devaney. Diffraction tomographic reconstruction from intensity data. IEEE Transactions on Image Processing, 1(2):221228, 1992.

[30] E. Candès, J. Romberg, and T. Tao. Robust uncertainty principles: Exact signal reconstruction from highly incomplete frequency information. IEEE Transactions on Information Theory, 52(2):489-509, 2006.

[31] Y. Wang, J. Yang, W. Yin, and Y. Zhang. A new alternating minimization algorithm for total variation image reconstruction. SIAM Journal on Imaging Sciences, 1(3):248-272, 2008.

[32] C. Li. An efficient algorithm for total variation regularization with applications to the single pixel camera and compressive sensing. PhD thesis, 2009.

[33] Yilun Wang, Junfeng Yang, Wotao Yin, and Yin Zhang. A new alternating minimization algorithm for total variation image reconstruction. SIAM Journal on Imaging Sciences, 1(3):248-272, 2008.

[34] A. Gonzalez-Ruiz, A. Ghaffarkhah, and Y. Mostofi. An integrated framework for obstacle mapping with see-through capabilities using laser and wireless channel measurements. IEEE Sensors Journal, 14(1):25-38, 2014.

[35] MobileRobots Inc. http://www.mobilerobots.com.

[36] Laird Technologies. http://www.lairdtech.com/Products/Antennas-and-Reception-Solutions/.

\section{APPENDIX}

Born Approximation: Consider the case of weak scatterers, where the electric properties of the objects in $\mathbf{D}$ are close to free space, i.e., $\epsilon(\mathbf{r})$ is close to $\epsilon_{0}$. In the Born approximation, this assumption is used to approximate the electric field inside the integral of (5) with $E_{\text {inc }}^{z}(\mathbf{r})$, resulting in the following approximation:

$$
E^{z}(\mathbf{r})=E_{\text {inc }}^{z}(\mathbf{r})+\iiint_{\mathbf{D}} G_{z z}\left(\mathbf{r}, \mathbf{r}^{\prime}\right)\left(O\left(\mathbf{r}^{\prime}\right) E_{\text {inc }}^{z}\left(\mathbf{r}^{\prime}\right)\right) d v^{\prime}
$$

The validity of the Born approximation is established by dimensional analysis in (5) and it is accurate at high frequencies, only if

$$
k_{0} L \delta_{\epsilon}(\mathbf{r}) \ll 1 \text {, for all } \mathbf{r} \in \mathbf{D},
$$

Born approximation is a theory of single scattering, wherein the multiple scattering due to object inhomogeneities is neglected. 


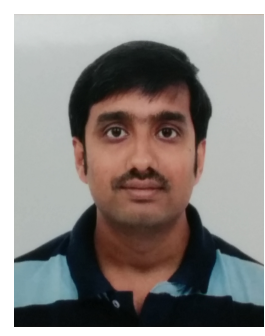

Saandeep Depatla received the Bachelors degree in Electronics and Communication Engineering from the National Institute of Technology, Warangal in 2010 and the MS degree in Electrical and Computer Science Engineering (ECE) from the University of California, Santa Barbara (UCSB) in 2014. From 2010 to 2012 he worked on developing antennas for radars in Electronics and Radar Development Establishment, India. Since 2013, he has been working towards his Ph.D. degree in ECE at UCSB. His research interests include signal processing, wireless communications and electromagnetics.

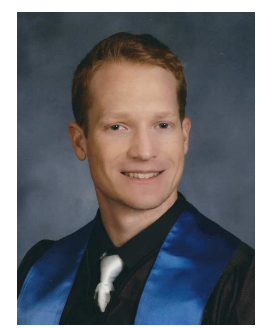

Lucas Buckland received his B.S. and M.S. degrees in Electrical and Computer Engineering from the University of California, Santa Barbara in 2013 and 2014 respectively. He is currently working as a research assistant in Professor Mostofi's lab at UCSB. His research interests include autonomous and robotic systems.

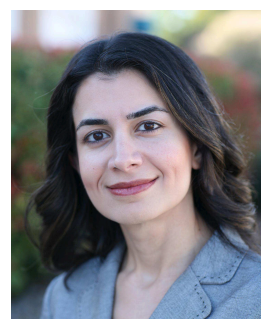

Yasamin Mostofi received the B.S. degree in electrical engineering from Sharif University of Technology, Tehran, Iran, in 1997, and the M.S. and Ph.D. degrees in the area of wireless communications from Stanford University, California, in 1999 and 2004, respectively. She is currently an associate professor in the Department of Electrical and Computer Engineering at the University of California Santa Barbara. Prior to that, she was a faculty in the Department of Electrical and Computer Engineering at the University of New Mexico from 2006 to 2012. She was a postdoctoral scholar in control and dynamical systems at the California Institute of Technology from 2004 to 2006. Dr. Mostofi is the recipient of the Presidential Early Career Award for Scientists and Engineers (PECASE), the National Science Foundation (NSF) CAREER award, and IEEE 2012 Outstanding Engineer Award of Region 6 (more than 10 western states). She also received the Bellcore fellow-advisor award from Stanford Center for Telecommunications in 1999 and the 2008-2009 Electrical and Computer Engineering Distinguished Researcher Award from the University of New Mexico. Her research is on mobile sensor networks. Current research thrusts include RF sensing, see-through imaging with WiFi, X-ray vision for robots, communication-aware robotics, and robotic networks. Her research has appeared in several news outlets such as BBC and Engadget. She has served on the IEEE Control Systems Society conference editorial board 2008-2013. She is currently an associate editor for the IEEE TRANSACTIONS ON CONTROL OF NETWORK SYSTEMS. She is a senior member of the IEEE. 